\section{Risk Factors for Cytomegalovirus Reactivation and Association With Outcomes in Critically Ill Adults With Sepsis: A Pooled Analysis of Prospective Studies}

\begin{abstract}
Hannah Imlay, ${ }^{1,2,3, \oplus}$ Sayan Dasgupta, ${ }^{2, \bullet}$ Michael Boeckh, ${ }^{1,2}$ Renee D. Stapleton, ${ }^{4}$ Gordon D. Rubenfeld, ${ }^{5}$ Ying Chen, ${ }^{2}$ and Ajit P. Limaye ${ }^{1}$

'Department of Internal Medicine, University of Washington, Seattle, Washington, USA, ${ }^{2}$ Vaccine and Infectious Disease Division, Fred Hutchinson Cancer Research Center, Seattle, Washington, USA, ${ }^{3}$ Department of Internal Medicine, University of Utah, Salt Lake City, Utah, USA, ${ }^{4}$ Larner College of Medicine, University of Vermont, Burlington, Vermont, USA, and ${ }^{5}$ Sunnybrook Health Sciences Center, Toronto, Canada
\end{abstract}

We performed multivariable analysis of potential risk factors (including cytomegalovirus [CMV] reactivation) for clinical outcomes by day 28 (death or continued hospitalization, ventilator-free days, intensive care unit (ICU)-free days, hospital-free days) from pooled cohorts of 2 previous prospective studies of CMV-seropositive adults with sepsis. CMV reactivation at any level, $>100 \mathrm{IU} / \mathrm{mL},>1000 \mathrm{IU} / \mathrm{mL}$, peak viral load, and area under the curve were independently associated with the clinical outcomes. We identified the potential effect size of CMV on outcomes that could be used as end points for future interventional trials of CMV prevention using antiviral prophylaxis in ICU patients with sepsis.

Keywords. cytomegalovirus; sepsis; mechanical ventilation; critical illness.

Cytomegalovirus (CMV) reactivation has been described in adults with critical illness caused by a broad range of etiologies, but especially bacterial sepsis. Multiple studies have demonstrated an association between CMV reactivation in critically ill adults and worse clinical outcomes, but have been limited by heterogeneous patient populations, inconsistent methodology across studies, and small numbers of patients [1]. Additional data are needed in order to rationally design phase 3 interventional trials that test the hypothesis that CMV causes poorer outcomes: specific virologic parameters that are associated with clinical outcomes, effect size of clinically relevant outcomes, and predictors of patient groups at high risk of CMV reactivation. The purpose of this study was to characterize specific CMV

Received 18 July 2020; editorial decision 28 October 2020; accepted 3 November 2020; published online November 6, 2020.

Correspondence: Hannah Imlay, MD, MS, University of Utah, 50 N Medical Drive, Salt Lake City, UT 84132 (hannah.imlay@hsc.utah.edu).

The Journal of Infectious Diseases ${ }^{\circledR} \quad$ 2021;223:2108-12

(C) The Author(s) 2020. Published by Oxford University Press for the Infectious Diseases Society of America. All rights reserved. For permissions, e-mail: journals.permissions@oup.com. DOI: 10.1093/infdis/jiaa697 reactivation parameters associated with clinical outcomes and to identify baseline risk factors that are associated with identified CMV reactivation parameters.

\section{METHODS}

\section{Patients and Study Design}

We performed secondary subgroup analyses of pooled data from 2 separate previously published prospective trials: cohort 1 , a subset of patients enrolled with sepsis from Limaye et al, 2008 [2] ( $n=40)$, a prospective observational study; and cohort 2 , a subset of patients enrolled with sepsis and who received placebo from Limaye et al, 2017 [3] $(n=66)$, a randomized, double blind, placebo-controlled phase 2 trial examining the effect of ganciclovir prophylaxis on outcomes of critical illness.

In these studies, adult CMV-seropositive patients with critical illness due to severe sepsis and associated respiratory failure and no known underlying or exogenous immunosuppression underwent prospective measurement of CMV DNAemia (using a previously published assay [4]) twice weekly until hospital discharge (cohort 1) or day 28 (cohort 2). Clinical outcomes were assessed at 28 days for both cohorts by study personnel who were blinded to CMV results. CMV reactivation was defined as detection CMV DNAemia by real-time polymerase chain reaction (PCR) in plasma.

\section{Variables}

Clinical outcomes included, by day 28: death or continued hospitalization, ventilator-free days (VFD), intensive care unit (ICU)-free days, and hospital-free days. VFDs, a previously developed parameter [5], were counted as 0 if the patient died before 28 days, VFDs $=28-\mathrm{x}$ if the patient was successfully weaned before day 28 (where $\mathrm{x}$ was the number of days requiring ventilation), and 0 if the patient required ventilation for $\geq 28$ days. ICU-free and hospital-free days were measured similarly.

CMV reactivation parameters included detectable DNAemia at any level, $>100 \mathrm{IU} / \mathrm{mL}$, and $>1000 \mathrm{IU} / \mathrm{mL}$, peak CMV load in $\log \mathrm{IU} / \mathrm{mL}$, and the average area under the curve (AUC) of CMV load in $\log \mathrm{IU} / \mathrm{mL}$ over the observed study period. The average AUC was calculated for each patient by summing all CMV PCR measurements and dividing by the number of days followed.

Demographic factors included age, race, sex, receipt of transfusion within 24 hours of admission, and a standardized Acute Physiology and Chronic Health Evaluation (APACHE) score. In the original studies, APACHE II (range 0-71) and APACHE III (range 0-299) scores were used; for the purposes of this study, a standardized APACHE score in each cohort was generated by 
subtracting the cohort mean from each observation in the cohort and dividing it by the cohort standard error. Absolute lymphocyte count (ALC) was collected at time of study enrollment in cohort 2. Transfusion status was defined as receipt of blood transfusion within the first 24 hours of hospital admission.

\section{Statistical Analysis}

Cumulative incidence of CMV reactivation at different viral load thresholds was calculated treating death as a competing risk.

To determine specific CMV parameters associated with the prespecified clinical outcomes, 20 multivariable logistic and linear models were performed. Potential confounders to be included in the model (age, race, sex, baseline transfusion status at enrollment, study cohort, and standardized APACHE score) were selected by backward stepwise regression. Each model examined the association between a measure of CMV reactivation and 1 of the clinical outcomes of interest.

To determine baseline risk factors associated with the above CMV parameters, 5 multivariable logistic and linear models were used to evaluate the association between demographic factors and CMV reactivation, adjusted for other demographic factors and study cohort.

Because ALC was collected only in cohort 2, a subset analysis restricted to this cohort examined the association between ALC and CMV reactivation. Logistic regression models were built and receiver operating characteristic (ROC) curves were generated to examine the relationship between ALC and CMV reactivation. From each ROC curve, the maximal value of Youden J statistic was used to identify the optimal model cutoff (that is, one that maximizes the sum of sensitivity and specificity) and, based on the inferred cutoff, an optimal ALC threshold was computed for characterizing each such relationship. In addition, ALC thresholds above which there was no CMV reactivation were assessed.

Multivariable models for each outcome were generated by incorporating all potential covariates as well as a covariate specific to each cohort. $P$ values $<.05$ were considered significant. Analyses were done in $\mathrm{R}$ version 3.5.1.

\section{RESULTS}

\section{Study Population and Incidence of CMV Reactivation}

One hundred and six patients were included in the combined cohort; demographics and clinical characteristics are shown in Table 1. The cumulative incidence of CMV reactivation at 0,100 , and $1000 \mathrm{IU} / \mathrm{mL}$ by 28 days was $41 \%$ (95\% confidence interval [CI], 32\%-53\%), 28\% (95\% CI, 20\%-39\%), and $18 \%$ (95\% CI, 11\%-28\%), respectively (Supplementary Figure 1). Patients from cohort 1 were only followed until hospital discharge; among the 45 patients in cohort 2 who were alive and discharged from the hospital before day 28 , only 1 patient (2.2\%) had new-onset CMV reactivation between hospital discharge and day 28.

\section{Clinical Outcomes}

Survival of the combined cohort was $86.8 \%$ (92/106) by 28 days. Among patients who survived to 28 days, $79.3 \%$ (73/92) were discharged from the hospital prior to 28 days. Median days of ventilator use, ICU stay, and hospital stay were 6 days

Table 1. Demographics and Clinical Outcomes of the Included Cohorts

\begin{tabular}{|c|c|c|}
\hline Characteristic & Cohort $1[2](n=40)$ & Cohort $2[3](n=66)$ \\
\hline Age, y, median (IQR) & 53.5 (47.0 to 62.3$)$ & 58 (51.5 to 67.0$)$ \\
\hline White race & $28(70.0)$ & $57(86.4)$ \\
\hline Sex male & $17(42.5)$ & $29(43.9)$ \\
\hline Transfusion within $24 \mathrm{~h}$ of hospital admission & $38(95.0)$ & $62(93.9)$ \\
\hline \multicolumn{3}{|l|}{ Study-specific APACHE score ${ }^{a}$} \\
\hline Mean & 26.9 & 77.56 \\
\hline Median (IQR) & $29.0(23.5$ to 31.3$)$ & 71.5 (51.5 to 95.8) \\
\hline \multicolumn{3}{|l|}{ Standardized APACHE score } \\
\hline Mean & 0 & 0 \\
\hline Median (IQR) & $0.28(-0.46$ to 0.58$)$ & $-0.21(-0.91$ to 0.64$)$ \\
\hline Mechanically ventilated & $29(72.5)$ & $66(100)$ \\
\hline Days inpatient before enrollment, mean & 0.25 & 3.15 \\
\hline \multicolumn{3}{|l|}{ Baseline ALC $C^{b}$} \\
\hline Mean & NA & 911.52 \\
\hline Median (IQR) & NA & $800(500$ to 1100$)$ \\
\hline Number still hospitalized at day 28 & $8(20)$ & $11(17)$ \\
\hline Number who died by day 28 & $4(10)$ & $10(15)$ \\
\hline
\end{tabular}

Data are No. (\%) except where indicated.

Abbreviations: ALC, absolute lymphocyte count; APACHE, Acute Physiology and Chronic Health Evaluation; IQR, interquartile range.

${ }^{a}$ APACHE II scores used in cohort 1, APACHE III scores used in cohort 2. A standardized APACHE score was created for this study so that the APACHE scores in each cohort were rescaled to have a mean of zero and a standard deviation of 1. The range for APACHE II scores is 0-71; the range for APACHE III scores is 0-299.

${ }^{\mathrm{b}} \mathrm{ALC}$ data only available for cohort 2 . 


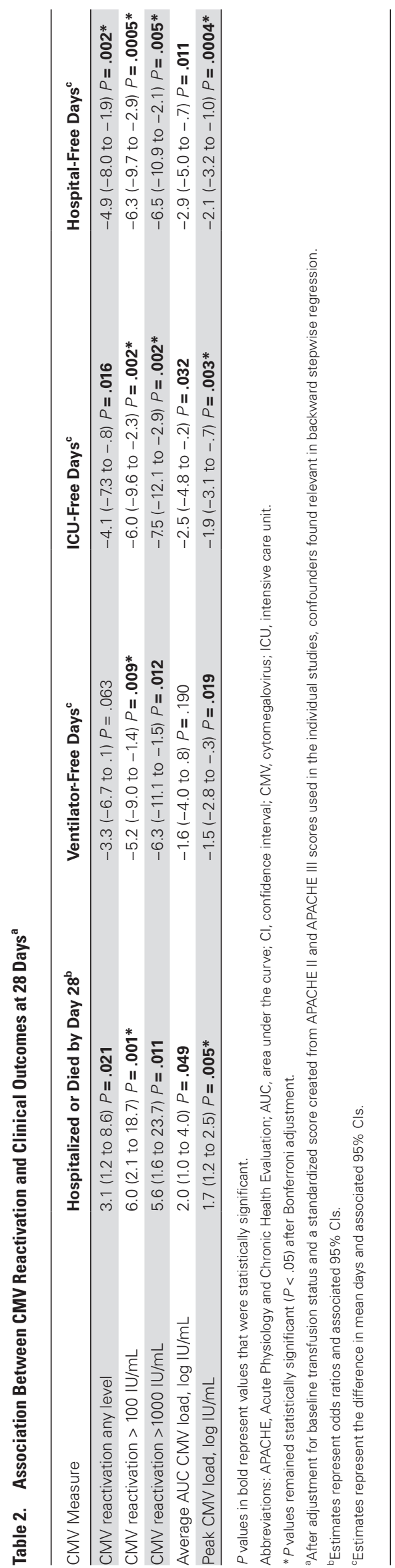

(interquartile range [IQR], 3-11), 8 days (IQR, 5-14), and 13 days (IQR, 8-22), respectively.

\section{Association of CMV Parameters With Clinical Outcomes}

The results of 20 multivariable models showing the association between CMV reactivation parameters and clinical outcomes at 28 days are shown in Table 2 (models adjusted for prior transfusion before enrollment and standardized APACHE score). Even after adjustment for potential confounding variables, various measures of CMV reactivation were significantly associated with each of the prespecified clinical outcomes. Because multiple measures of $\mathrm{CMV}$ reactivation were examined, Bonferroni adjustment was performed for the $P$ values of each model.

\section{Association of Baseline Demographic Variables With CMV Reactivation}

Age, race, sex, or transfusion within 24 hours of admission was not associated with any CMV reactivation parameter $(P>.05$ for all comparisons; Supplementary Table 1). The standardized APACHE score was inconsistently associated with certain CMV reactivation measures: $>100 \mathrm{IU} / \mathrm{mL}$ (odds ratio [OR], 1.6; 95\% CI, $1.0-2.7 ; P=.049$ ) and average AUC of CMV load (1 unit increase in standardized APACHE score associated with 0.2 higher log AUC of CMV viral load [95\% CI, .01-.3; $P=.04]$ ), but not other CMV measures.

In adjusted models using data from cohort 2, the median ALC was 800 (IQR, 500-1100). ALC was significantly associated with CMV reactivation at any level $(P=.02)$ and peak CMV load $(P=.02)$, but not with other measures of CMV reactivation. The area under the ROC curves associated with CMV reactivation at any level, CMV reactivation $>100 \mathrm{IU} / \mathrm{mL}$, and CMV reactivation $>1000 \mathrm{IU} / \mathrm{mL}$ were $0.653,0.659$, and 0.671 , respectively; there were no specific threshold ALC values that predicted CMV reactivation at these thresholds with high negative or positive predictive values (Supplementary Figure 2). The calculated optimal ALC values associated with CMV reactivation at any level, CMV reactivation $>100 \mathrm{IU} / \mathrm{mL}$, and CMV reactivation $>1000 \mathrm{IU} / \mathrm{mL}$ were associated with low sensitivity and specificity (Supplementary Table 2). At a baseline ALC threshold of $>1400$ cells/ $\mu \mathrm{L}$ (10 of $65,15 \%$ ), none developed CMV reactivation (Supplementary Table 3).

\section{DISCUSSION}

This study used pooled data from prior prospective cohorts to identify and refine specific CMV parameters associated with worse clinical outcomes and to identify baseline demographic factors that could be used to "enrich" a patient population for a phase 3 clinical trial of CMV prevention to improve outcomes in the ICU setting.

In adjusted models, multiple measures of CMV reactivation were associated with various clinical outcomes and there was a quantitative relationship (ie, the higher the viral load the greater the association with the clinical outcome). Notably, CMV 
reactivation at any level was associated with worse outcomes, providing support for a preventative (prophylaxis) antiviral strategy over a test and treat (preemptive) approach for initial efficacy studies designed to assess whether CMV suppression improves clinical outcomes.

Previous studies have suggested that severity of illness may confound the relationship between CMV reactivation and worse clinical outcomes [6,7]; however, not all studies have found this association $[1,2]$. In this study, APACHE score was used as a proxy for severity of illness and was inconsistently associated with most measures of CMV reactivation. After adjustment for baseline APACHE score, multiple measures of CMV DNAemia remained independently associated with worse clinical outcomes, consistent with previous findings $[8,9]$. These data are consistent with the hypothesis that even though severity of illness might increase the risk for CMV reactivation, CMV reactivation may still independently contribute to worse clinical outcomes, although the specific mechanisms remain undefined. Only carefully conducted interventional trials can definitively distinguish between a causal versus marker role of CMV in this clinical setting.

We also assessed other baseline characteristics and did not identify patient subgroups at higher risk for CMV reactivation that could be specifically targeted for future interventional trials. Studies performed in other patient populations have suggested that lymphopenia is a predictor of CMV reactivation [10-13]; in our study, using ROC analysis, we did not identify a specific baseline ALC threshold that was significantly associated with measures of CMV. However, we identified a relatively high ALC threshold (>1400/ $\mu \mathrm{L})$ above which CMV reactivation did not develop. If confirmed in larger studies, this criterion could potentially be used to exclude patients with low risk of CMV reactivation. Lymphocyte count and other immune parameters that predict CMV reactivation [14] should be investigated further. Measurements of CMV-specific immunity also should be further investigated as predictors for CMV reactivation in this setting, as previously reported [15].

Strengths of this study include the prospective multicenter design with regularly monitored CMV parameters, study personnel blinded to CMV results, and prespecified clinical outcomes. Our study was limited by clinical variables collected as part of the primary studies; however, the covariates we used had previously been identified as factors associated with poor outcomes or CMV reactivation. We performed multiple analyses, increasing the risk for false associations. However, the observed associations were seen in 2 independent cohorts [2,3], and in 2 separate metaanalyses of CMV reactivation and clinical outcomes in ICU patients $[1,6]$, making it less likely that the observed associations were spurious. Lastly, our sample size was moderate; however, this represents the largest cohort to date in which CMV reactivation and outcomes were tracked prospectively.
Multiple studies have shown an association between CMV DNAemia among adults with sepsis and worse clinical outcomes-however, adequately powered phase 3 trials are needed to distinguish between a causal versus marker relationship. The relatively high rate of $\mathrm{CMV}$ reactivation, combined with the magnitude of association with several clinically relevant outcomes, supports the feasibility of an efficacy study of CMV prevention to improve clinical outcomes in CMV-seropositive adults with sepsis.

\section{Supplementary Data}

Supplementary materials are available at The Journal of Infectious Diseases online. Consisting of data provided by the authors to benefit the reader, the posted materials are not copyedited and are the sole responsibility of the authors, so questions or comments should be addressed to the corresponding author.

\section{Notes}

Acknowledgment. We gratefully acknowledge Lili Peng, Yixin Wang, and $\mathrm{Hu}$ Xie for assistance with data management.

Disclaimer. The funding source had no input in study design, analysis, interpretation of results, nor the decision to submit for publication

Financial support. This work was supported by the National Institutes of Health/National Institute of Allergy and Infectious Diseases (grant numbers T32 AI118690-03 to H. I.; R56 AI140953 to Y. C.; T32 AI118690-03, K24 HL09329410, and U01 HL102547-01 to M. B.; HHSN272201600016C, HHSN272201100041C, and HHSN272201600019C to A. P. L.); and by an investigator-initiated research grant from Merck to A. P. L.

Potential conflicts of interest. H. I. reports support as a site investigator for Gilead and Janssen. G. D. R. reports support as an advisor to Endpoint Health. M. B. reports consulting fees from Allovir; consulting fees and research support from Gilead; consulting fees and research support from Merck; consulting fees, stock ownership, and advisory board member for Helocyte; consulting fees, share options, advisory board member for EvrysBio; consulting fees from GSK; consulting fees from SymBio; research support from Lophius Biosciences; consulting fees and research support from Shire/Takeda; and consulting fees from Moderna. A. P. L. reports receipt of grant funding from Merck as above. All other authors report no potential conflicts.

All authors have submitted the ICMJE Form for Disclosure of Potential Conflicts of Interest. Conflicts that the editors consider relevant to the content of the manuscript have been disclosed.

Presented in part: IDWeek, 2-6 October 2019, Washington, DC. 


\section{References}

1. Lachance P, Chen J, Featherstone R, Sligl WI. association between cytomegalovirus reactivation and clinical outcomes in immunocompetent critically ill patients: a systematic review and meta-analysis. Open Forum Infect Dis 2017; 4:ofx029.

2. Limaye AP, Kirby KA, Rubenfeld GD, et al. Cytomegalovirus reactivation in critically ill immunocompetent patients. JAMA 2008; 300:413-22.

3. Limaye AP, Stapleton RD, Peng L, et al. Effect of ganciclovir on IL-6 levels among cytomegalovirus-seropositive adults with critical illness: a randomized clinical trial. JAMA 2017; 318:731-40.

4. Boeckh M, Huang M, Ferrenberg J, et al. Optimization of quantitative detection of cytomegalovirus DNA in plasma by real-time PCR. J Clin Microbiol 2004; 42:1142-8.

5. Schoenfeld DA, Bernard GR; ARDS Network. Statistical evaluation of ventilator-free days as an efficacy measure in clinical trials of treatments for acute respiratory distress syndrome. Crit Care Med 2002; 30:1772-7.

6. Kalil AC, Florescu DF. Prevalence and mortality associated with cytomegalovirus infection in nonimmunosuppressed patients in the intensive care unit. Crit Care Med 2009; 37:2350-8.

7. Walton AH, Muenzer JT, Rasche D, et al. Reactivation of multiple viruses in patients with sepsis. PloS One 2014; 9:e98819.
8. Heininger A, Haeberle H, Fischer I, et al. Cytomegalovirus reactivation and associated outcome of critically ill patients with severe sepsis. Crit Care 2011; 15:R77.

9. Ong DSY, Spitoni C, Klein Klouwenberg PMC, et al. Cytomegalovirus reactivation and mortality in patients with acute respiratory distress syndrome. Intensive Care Med 2016; 42:333-41.

10. Papazian L, Hraiech S, Lehingue S, et al. Cytomegalovirus reactivation in ICU patients. Intensive Care Med 2016; 42:28-37.

11. Chiche L, Forel JM, Thomas G, et al. Interferon- $\gamma$ production by natural killer cells and cytomegalovirus in critically ill patients. Crit Care Med 2012; 40:3162-9.

12. Travi G, Pergam SA. Cytomegalovirus pneumonia in hematopoietic stem cell recipients. J Intensive Care Med 2014; 29:200-12.

13. Gardiner BJ, Nierenberg NE, Chow JK, Ruthazer R, Kent DM, Snydman DR. Absolute lymphocyte count: a predictor of recurrent cytomegalovirus disease in solid organ transplant recipients. Clin Infect Dis 2018; 67:1395-402.

14. Saldan A, Forner G, Mengoli C, Gussetti N, Palù G, Abate D. Strong cell-mediated immune response to human cytomegalovirus is associated with increased risk of fetal infection in primarily infected pregnant women. Clin Infect Dis 2015; 61:1228-34.

15. Castón JJ, Cantisán S, González-Gasca F, et al. Interferon- $\gamma$ production by $\mathrm{CMV}$-specific $\mathrm{CD} 8^{+} \mathrm{T}$ lymphocytes provides protection against cytomegalovirus reactivation in critically ill patients. Intensive Care Med 2016; 42:46-53. 